\title{
The Effect of Individual's Influence Power on Consensus Emergency Model
}

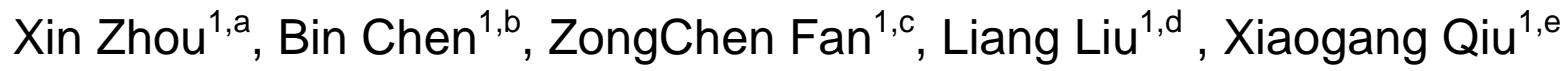 \\ ${ }^{1}$ College of Information and Management, National University of Defense Technology, Changsha, \\ 410073, China. \\ aemail: zhouxinnudt@163.com, bemail: \\ nudtcb9372@gmail.com, cemail:andy_van1@sohu.com, ${ }^{d}$ email:nudt_II@163.com, eemail:1387493 \\ 4509@139.com
}

Keywords: Consensus Emergency Model; Influence Power; Behavior Mechanism; Simulation

\begin{abstract}
Aiming at deficiencies of previous researches on consensus emergency model, this paper provides a new consensus emergency model. In the first place, we extract individual's influence power as its main character based on social psychology. Furthermore, the behavior mechanism of individual is constructed. According to the model of individual, a group of agents whose relationship is small word network is built. By simulating a phenomenon which people who are unaware of the truth are deluded by rumormongers in XinJiang $7 \cdot 5$ Incident, we get a possible process that rumors spread among the crowd of Uyghur people. The simulation results show that the research work plays an important role in opinion monitoring and intervention.
\end{abstract}

\section{Introduction}

Consensus refers to the certain tendency of comments, opinions or attitudes towards a particular event of most people [1]. There are two research methods on consensus currently. One method is analyzing the data crawled from Internet. The other method is constructing individual model based on multi-agent theory.

Analysis of network consensus is a systematic project, which involves many technologies. Such as web page collection technology, text representation model, lexical analysis, text clustering, text classification and text orientation analysis etc. Many application researches can be done based on these technologies.

Based on multi-agent theory, individual is regarded as an agent and each agent has its own properties and behaviors. There are many researches on consensus emergency model based on the theory. Among them, some models excavate the substantive characteristics of consensus to provide the theoretical basis of the evolution of consensus. Such as Sznaid Model [2], Bounded Confidence Model [3][4] and Galam Model [5][6] etc. Some other models are put forward based on the former achievements.

In this paper, we provide a model of consensus emergency model based on multi-agent theory. This model extracts the characteristics and behavior mechanisms of individual. Through simulating the model, we get a propagative process of rumor of crowd and the varied trend of the whole crowd's opinion.

\section{Modeling of the Individual's Character}

An individual is modeled as an agent, and the network society consists of a number of agents. Agent can be abstracted as index, opinion, event role, stubborn and influence power. Index presents the ID of agent. Each agent has only one ID which is different from others. Opinion is the attitude towards information. Each agent plays a role in a certain event. We call it event role. A small group of agents are easy to attract the attentions from others. Their opinions always have a significant influence on others. Most of agents are hard to attract other's attention. Their opinions have low influence on others. So we divide event roles into two categories: opinion leader and ordinary netizen. Influence power is the ability which individual persuade others. However, in the process of 
interaction, it is not equal between two individuals. If someone has higher qualification, elder age, higher status and more knowledge, he may persuade other more easily and vice versa. Stubborn is the attitude of rejecting other's opinion. Everyone would like his opinion to be accepted by other person. So, people will reject other's opinion to some degree.

\section{Modeling of the Individual's Behavior}

When a hot spot occurs, a few active netizens will focus on this hot spot the first time. They will process the information according to their own needs, and then spread it to other netizens. Roughly speaking, we can call them opinion leader. After that, the information will be spread and discussed in substantial number of netizens.

For the convenience of descripting the model, we use agent $i$ as the object of study. Figure 1 shows the interaction between two agents.

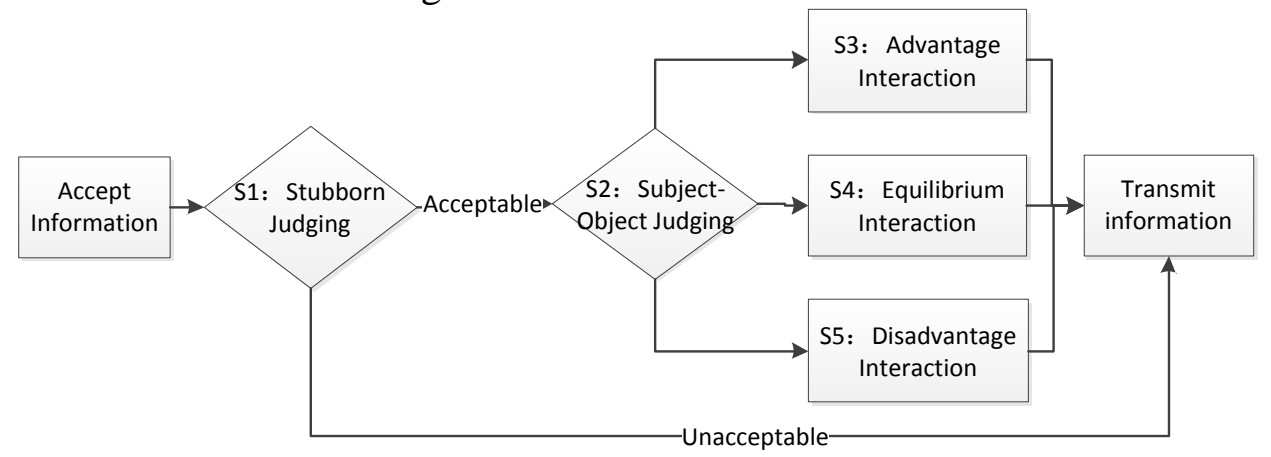

Fig. 1. Communicating with the other agents.

Step 1: Stubborn Judging

When communicating with other agents, agent $i$ will compare the opinion between the opposite and itself. If the divergence of opinion is acceptable, they will exchange idea further. If the divergence of opinion is unacceptable, they will not change their opinion and only know the information.

The algorithm is: If $\left(\left|O_{i}(t)-O_{j}(t)\right|<\left(1-S_{i}(t)\right)\right)$, Then enter Step2; Else exit. Where $O_{i}(t)$ is the opinion of agent $i ; O_{j}(t)$ is the opinion of agent $j ; S_{i}(t)$ is the stubborn of agent $i$; $t$ represent the simulation time is at the moment.

$>\quad$ Step2: Subject-Object Judging

Three situations may occur. Influence power of agent $i$ is higher than the other; Influence power of agent $i$ is equal to the other; Influence power of agent $i$ is lower than the other.

The algorithm is: If $\left(I_{i}(t)-I_{j}(t)>\alpha\right)$, Then enter Step3; Else If $\left(\left|I_{i}(t)-I_{j}(t)\right|<\alpha\right)$, Then enter Step4; Else If $\left(I_{j}(t)-I_{i}(t)>\alpha\right)$, Then enter Step5.

Where $I_{i}(t)$ is the influence of agent $i, I_{j}(t)$ is the influence power of the agent $j$ and $\alpha$ is an adjustable parameter which is greater than 0 .

\section{$>\quad$ Step3: Advantage Interaction}

In this situation, agent $i$ won't change its opinion completely. The formula is as follow.

$O_{i}(t+1)=O_{i}(t)$

Where $O_{i}(t)$ and $O_{i}(t+1)$ are the opinion of agent $i, t$ presents the simulation time is at the moment.

$>\quad$ Step4: Equilibrium Interaction

In this situation, the strength of both sides is equality. So, agent $i$ will change its opinion to some degree. The formula is as follow.

$$
O_{i}(t+1)=O_{i}(t)+I_{j}(t)\left(1-S_{i}(t)\right)\left(O_{j}(t)-O_{i}(t)\right)
$$

Where $O_{i}(t)$ and $O_{i}(t+1)$ are the opinion of agent $i, I_{i}(t+1)$ and $I_{i}(t)$ are the influence power of agent $i ; I_{j}(t)$ presents the influence power of agent $j$; $S_{i}(t)$ presents the stubborn of agent $i$; $t$ presents the simulation time is at the moment.

$>\quad$ Step5: Disadvantage Interaction 
In this situation, agent $i$ will change its opinion completely. The formula is as follow.

$O_{i}(t+1)=O_{j}(t)$

Where $O_{i}(t+1)$ are the opinion of agent $i, O_{j}(t)$ presents the opinion of agent $j$, and $t$ presents the simulation time is at the moment.

\section{Simulation Experiment}

In recent years, the number of terrorist attack incidents is persistent growth in China. The XinJiang 7.5 Incident is one of the most hazardous incidents. There is a phenomenon that many perpetrators are unaware of the truth, and they just follow what their friends do in the incident. So we simulate a case that how ordinary people are persuaded by rumormongers.

In this experiment, there are two types of people, one is ordinary people and the other is rumormonger. Ordinary people are considered as ordinary netizen and rumormonger as opinion leader. The aim of opinion leaders is to persuade ordinary netizen to accept their opinions. Based on the background of the case, we simulate rumors spread in 10 thousand agents whose relationship is a small-world network.

$>\quad$ Specifying the value range of parameters

For the convenience of descripting the model, we use agent $i$ as the object of study.

The opinion is denoted by $O_{i}$. We define $O_{i} \in[0,1]$ and $O_{i} \in \mathrm{R}$. For the convenience of analyzing, we divide $O_{e}$ into five categories: completely accept, slightly accept, neutrality, slightly opposite and completely opposite. The opinion can be abbreviated as follow: $a=$ complete accept, $a \in[0.8,1]$; $b=$ slightly accept, $b \in[0.6,0.8) ; c=$ neutrality, $c \in[0.4,0.6) ; d=$ slightly opposite, $d \in[0.2,0.4) ; e=$ complete opposite, $e \in[0,0.2)$.

The event role is denoted by $E_{i}$. It includes opinion leader and ordinary netizen.

The influence power is denoted by $I_{i}$. We define $I_{i} \in[0,1]$ and $I_{i} \in \mathrm{R}$. The upper limit of $I_{i}$ presents the highest influence power; the lower limit of $I_{i}$ presents the lowest influence power.

The stubborn is denoted by $S_{i}$. We define $S_{i} \in[0,1]$ and $S_{i} \in \mathrm{R}$. The upper limit of $S_{i}$ presents the highest stubborn; the lower limit of $S_{i}$ presents the lowest stubborn.

Assignment of agent

Firstly, we set the proportion of opinion leaders. We assume that the proportion of opinion leaders is 0.1 in this case, who are randomly assigned to any node in the network.

For the sake of their profit, opinion leaders accept rumor completely. Opinion leaders have background knowledge. It is easy for opinion leaders to persuade ordinary netizens who don't know the truth. Hence, their influence power should be high. Because the aim of opinion leader is to persuade others, it is hard to be persuaded by the other people. On the other hand, ordinary netizens may not accept these rumors at the initial time. However, most ordinary netizens will not investigate rumors, and have little background knowledge about it. We assume ordinary netizens will accept the opinion which is different from theirs easily. Hence, the assignments of agents are listed as follow.

Table. 1. Assignment of agent's characters.

\begin{tabular}{ccc}
\hline Event role & Opinion leader & Ordinary netizen \\
\hline Number & 999 & 9001 \\
Opinion & $\mathrm{U}[0.8,1]$ & $\mathrm{U}[0,0.8]$ \\
Influence Power & $\mathrm{U}[0.8,1]$ & variable \\
Stubborn & $\mathrm{U}[0.4,0.6]$ & $\mathrm{U}[0.1,0.3]$
\end{tabular}

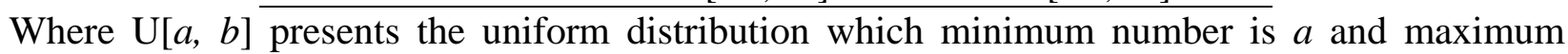
number is $b$.

\section{$>\quad$ Simulation results}

In this experiment, we set the influence power of agent as variable. We do four experiments which the influence of ordinary netizens is in interval [0,0.1] $[0.1,0.2],[0.2,0.3]$ and $[0.3,0.4]$.

Figure 2 shows the experiment result. Figure 2 (a) shows the change of number of agents who completely accept the rumor. Figure 2 (b) shows the change of number of agents who slightly accept rumor. The abscissa represents simulation iterations, and simulation forward $t=1$ unit each 
step. The vertical axis represents the number of agent. Each picture has four curves which present the influence of ordinary netizens is in interval [0,0.1] $[0.1,0.2],[0.2,0.3]$ and $[0.3,0.4]$ respectively.

As figure 2 (a) and (b) shown, if the influence power of ordinary netizen is generally low, they are easily persuaded by opinion leader, while if the influence power of ordinary netizen exceeds a certain threshold, it is hard to persuade them.

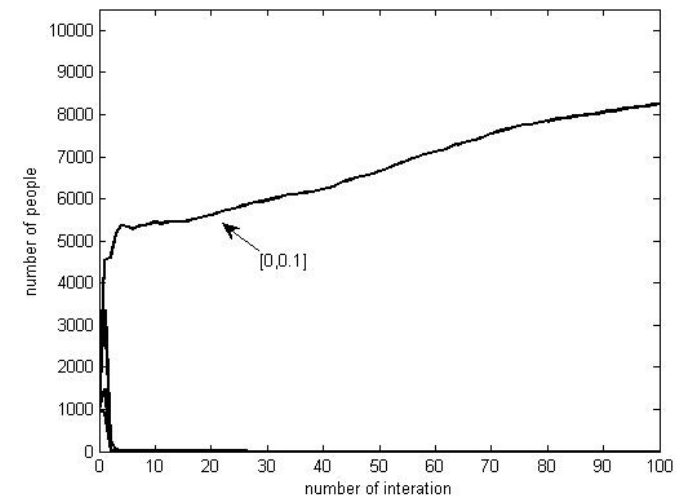

(a) Agents who completely accept rumor.

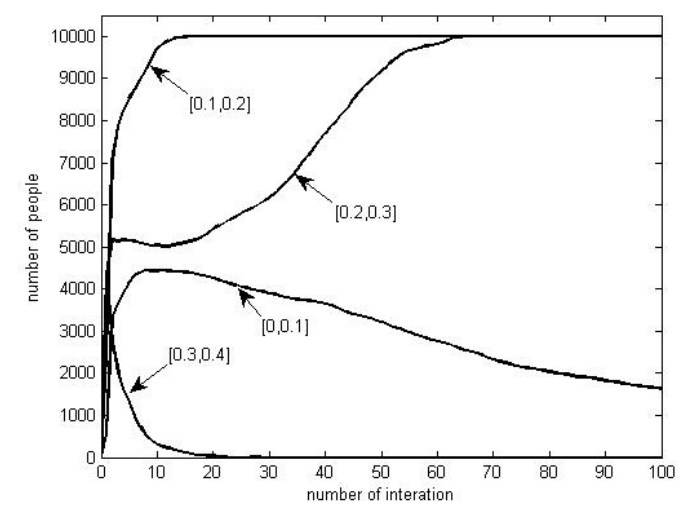

(b) Agents who slightly accept rumor.

Fig. 2. The experimental results of influence power of ordinary netizens where $\alpha=1$.

\section{Conclusion}

Through the simulation, we can know the reason that many perpetrators follow what their friends do in XinJiang 7.5 Incident may be the lower influence power of crowd. Consequently, if we intend to prevent the persistent propagation of rumor, the influence power which means the ability of distinguishing the true from the false of crowd should be improved.

\section{Acknowledgement}

In this paper, the research was supported by National Nature and Science Foundation of China under Grant (Nos. 91024030 and 91324013).

\section{References}

[1] LIU Chang-yu, HU Xiao-feng, LUO Pi, SI Guang-ya. Study on Consensus Emergency Model Based on Asymmetric Personal Relationship Influence[J]. Journal of System Simulation,2008.2,20(4):990-996.

[2] Sznajd Weron K, Sznajd J. Opinion Evolution in Closed Community[J]. International Journal of Modern Physics C, 2000, 11(6): 1157-1165.

[3] Deffuant G, NeauD, Amblard F, et al. Mixing Beliefs Among Interacting Agents[J]. Advances in Complex Systems, 2000, 3: 87-98.

[4] Hegselmann R, Krause U. Opinion Dynamics and Bounded Confidence Models, Analysis, and Simulations[J]. Journal of Artificial Societies and Social Simulation, 2002, 5(3):1-33.

[5] Galam S. Minority Opinion Spreading in Random Geometry[J]. Eur. Phys. J. B, 2002, 25(4): 403-406.

[6] Galam S. Sociophysics: A Review of Galam Models[J].International Journal of Modern Physics C, 2008, 19(3):409-440. 